\title{
RVG-Mediated Calpain2 Gene Silencing in the Brain Impairs Learning and Memory
}

\author{
Sohila Zadran • Garnik Akopian • Homera Zadran • \\ John Walsh • Michel Baudry
}

Received: 10 July 2012/ Accepted: 3 August 2012/Published online: 19 August 2012

(C) Springer Science+Business Media, LLC 2012

\begin{abstract}
In the central nervous system, two calpain isoforms are highly expressed: calpain1 and calpain2. Here, we show for the first time that activation of the calpain isoform, calpain2, is a necessary event in hippocampal synaptic plasticity and in learning and memory. We developed a fluorescence resonance energy transfer-based animal model to monitor in vivo calpain activation in single cells and in real time. Additionally, utilizing a novel rabies virus glycoprotein-chimeric peptide, which enabled the transvascular delivery of small interfering RNA to the brain against calpain2, we down-regulated the calpain2 isoform in vivo. Calpain2 gene silencing eliminated longterm potentiation and impaired learning and memory. Our results not only identify the calpain2 isoform as a critical mediator in learning and memory but also highlight an innovative, highly efficient calpain2-targeting peptide capable of isoform-specific gene silencing in the brain. We anticipate these innovative technologies and our better understanding of the calpain machinery, particularly of the calpain2 isoform, will have substantial influence on future translational studies, attracting considerable interest in the use of calpain models and calpain-specific inhibitors in the development of therapeutics.
\end{abstract}

Electronic supplementary material The online version of this article (doi:10.1007/s12017-012-8196-8) contains supplementary material, which is available to authorized users.

S. Zadran $(\bowtie)$

Division of Biology, California Institute of Technology,

1200 East California Blvd, Pasadena, CA 91125, USA

e-mail:szadran@mednet.ucla.edu

G. Akopian · H. Zadran · J. Walsh · M. Baudry

Department of Biological Sciences, University of Southern

California, 3641 Watt Way, HNB 514, Los Angeles, CA 90089,

USA
Keywords Calpain $\cdot$ Synaptic plasticity $\cdot$ Rabies virus glycoprotein · Learning and memory

\section{Introduction}

Calpains are ubiquitous calcium-dependent, neutral proteases and are unique in that they usually do not degrade their target proteins, but instead modify their functions by partial proteolysis (Goll et al. 2003; Liu et al. 2008). In the central nervous system (CNS), two calpain isoforms are highly expressed: calpain1 and calpain2. Calpain1 and calpain2 (also known as $\mu$-calpain and $\mathrm{m}$-calpain, respectively) have similar physiological functions, but differ in the calcium concentrations required for activation, with calpain1 requiring $1-20 \mu \mathrm{M}$ of calcium and calpain2 requiring between 0.25 and $0.75 \mathrm{mM}$ of calcium for activation (Chan et al. 1999).

Thirty years ago, we first proposed neuronal calpains as a critical regulator of synaptic plasticity and learning and memory (Zadran et al. 2010a; Lynch et al. 1984). Recent studies have implicated neuronal calpains (calpain1 and calpain2) to specifically target a large variety of postsynaptic substrates, including spectrin and cortactin, postsynaptic density proteins, such as PSD-95 and SAP97, calcium- and calmodulin-dependent protein kinase II (CaMKII), and the AMPA, NMDA and mGluR1 glutamate receptors (Sorimachi et al. 2011; Czogalla et al. 2005; Demarchi et al. 2007; Greget et al. 2011; Vosler et al. 2008; Verity 1992). However, calpain isoform-specific substrates and isoform-specific functions have yet to be determined, in large part due to the limitations of present technologies.

Currently, there are no specific inhibitors for the various calpain isoforms, and even the available calpain inhibitors 
are not entirely specific for this class of proteases (Donkor 2011). Knockout of calpain isoforms has also proven to be problematic as well. While calpain 2 knockout is embryonic lethal, calpain1 knockout mice did not exhibit the obvious neuronal phenotype (Grammer et al. 2003; Takano et al. 2011). Thus, there is a clear need to develop new technologies in order to monitor both in vitro and in vivo calpain processes and calpain isoform-specific functions in the brain. Here, we develop a FRET-based animal model to monitor in vivo calpain activation in real time in single cells. Utilizing this animal model, we were able to monitor differential calpain activation in single cells in various brain regions in real time. We also synthesized an RVGtargeting peptide to transiently down-regulate calpain2 in the brain to determine the isoform-specific functions of neuronal calpain2. These technologies identified for the first time that calpain 2 activation is a necessary event in synaptic plasticity and learning and memory. We anticipate these innovative technologies and our better understanding of the calpain machinery, particularly of the calpain2 isoform, will have substantial influence on future translational studies. Abnormal calpain function has already been implicated in several pathologies, including stroke, neurodegeneration, and Alzheimer's disease (Camins et al. 2006; Huang et al. 2001), fueling the need for innovative calpain models and calpain-specific inhibitors, particularly for the development of therapeutics.

\section{Materials and Methods}

\section{Generation of CAFI Mice}

Calpain activity monitored by FRET imaging (CAFI) mice was a generous gift from Dr. Isabelle Richard (Généthon). Briefly, CAFI mice were generated by Speedy Mouse ${ }^{\circledR}$ Technology (Nucleis, France) to produce a single-copy transgene insertion at the hprt locus. The coding sequence of the calpain sensor was excised as a NheI-AflII fragment and cloned into the EcoRI site of the polylinker of the Gateway ${ }^{\circledR}$ pENTR-1A vector (Invitrogen) previously modified to include the ubiquitous cytomegalovirus immediate-early enhancer chicken $\beta$-actin hybrid (CAG) promoter. The transgene was then transferred by the Gateway technology into a destination vector pDESTHPRT (Nucleis) carrying two homologous arms of the hprt gene. The vector was linearized using PvuI and electroporated into hprt-deficient BPES-embryonic stem (ES) cells by standard methods. The targeting construct contained the missing sequences in BPES-ES cells and two regions of homology of the hprt gene, which allowed insertion of the transgene at this locus. The targeted ES clones were efficiently selected, thanks to the restoration of the ability to grow in hypoxanthine-aminopterin-thymidine (HAT) medium through a correct homologous recombination. Targeted ES cells were injected into C57BL/6derived blastocysts that were then transplanted into the uteri of recipient females. Resulting chimeric males were bred with C57BL/6 females, and the F1 agouti female offspring were backcrossed with C57BL/6 males. CAFI mice were genotyped by visualization of the fluorescence of the tail sample after YFP excitation.

\section{Acute Hippocampal Slices}

Acute hippocampal slices were isolated as previously described (Zadran et al. 2009, 2010b).

\section{FRET Fluorescence Quantification and FRET Imaging}

FRET laser scanning confocal images were collected on a Nikon Eclipse TE-200 equipped with argon-ion blue lasers $(457 \mathrm{~nm})$. Analysis was performed on ImageJ. FRET signal was also quantified via spectrofluorometer using CFP excitation and emission wavelengths $(\lambda=408 \mathrm{~nm}$ and $\lambda=480 \mathrm{~nm}$, respectively).

Filamentous Actin Staining and Actin Polymerization Assay

Actin polymerization was quantified by a rhodaminephalloidin fluorescence enhancement assay (Zadran et al. 2009, 2010b). Real-time actin polymerization in acute hippocampal slices was monitored as acute slices were topically treated with $5 \mu \mathrm{M}$ Alexa594-phalloidin (Invitrogen) and subjected to confocal microscopy.

\section{Western Blotting}

Western blotting was conducted as previously described (Zadran et al. 2009, 2010b). Antibodies against the following proteins were used at the indicated concentrations: calpain2 (AB81023; 1:1,000 dilution, Millipore Corporation) and actin (MAB1501 1:5,000, Millipore Corporation). Anti- $\beta$-actin was used as loading control, and results were adjusted against actin signal.

Drug Treatments

CAFI or C57BL/6 samples were treated with calpain inhibitor III $(10 \mu \mathrm{M})$, before treatment with BDNF $(50 \mathrm{ng} / \mathrm{ml})$ or TEA $(10 \mathrm{mM})$ for indicated time periods prior to FRET or biochemical analysis. 
Electrophysiology

Electrophysiology was conducted as previously described (Chen et al. 2007). Briefly, acute hippocampal slices or cultured hippocampal neurons were placed in a holding chamber for at least $1 \mathrm{~h}$ prior to being transferred to a recording chamber while submerged in aCSF. CA1 pyramidal neurons or single cells were visualized using confocal microscopy (Nikon). Whole-cell recordings were made with 3-5 $\mathrm{M} \Omega$ recording pipettes containing the following (in mM): $130 \mathrm{Cs}$ gluconate, $10 \mathrm{CsCl}, 0.2$ EGTA, 8 $\mathrm{NaCl}, 2$ ATP, 0.3 GTP, and 10 HEPES, pH 7.35, 290-300 mOsm. Baseline responses were collected for $15 \mathrm{~min}$, after which theta burst stimulation (TBS), five bursts of four stimuli delivered at $100 \mathrm{~Hz}$ repeated at $5 \mathrm{~Hz}$, was applied through one of the two stimulation electrodes. Samples were then subjected to analysis. Hippocampal slices were microdissected for CA1 prior to analysis. For stimulation in cultured neurons, neurons were stimulated with a homogenous electrical field generated by two parallel platinum wires ( $7 \mathrm{~mm}$ long, distance of $7,1 \mathrm{~mm}$ distance from cells). A similar TBS pattern was used.

\section{RVG Peptide Synthesis}

A RVG-bound calpain2 siRNA peptide was synthesized as follows: RVG (YTIWMPENPRPGTPCDIFTNSRGK RASNG) was purchased from Anaspec and coupled to nine D-arginine allowing calpain2 siRNA complex formation [siRNA sequence: calpain2, 5'-UUCGGCUGAAUGCA CAAAGAGCAGC-3'] . 6-8 weeks old C57Bl/6 mice were injected in the tail vein with the RVG peptide constructs (50 $\mu \mathrm{g}$ of calpain2 per mouse per injection) prepared in $100 \mu \mathrm{l}$ of $5 \%$ glucose. Mice were injected daily for 4 days prior to behavioral assays.

\section{Cytotoxicity Assay}

To test the cytotoxicity of the RVG peptide complex, primary hippocampal neurons were treated with the complex for $48 \mathrm{~h}$ before viability was determined with a MTT Cell Growth Assay Kit (Millipore).

\section{Fear Conditioning}

Fear-conditioning behavioral analysis was performed as described by Clausen et al. (2007). All behavior was observed and analyzed with the experimenter blind to the conditions: wild-type or CAFI animals. Behavior was recorded with a video camera that was linked to a TV monitor and VCR for online observation and recording. On day 1 , mice were individually placed in a $10.5 \times 12 \times 12^{\prime \prime}$ conditioning chamber with electrifiable flooring (Coulbourn
Instruments). To enhance the salience of the chamber, three walls had broad, 1-in., red-and-white diagonal stripes at $45^{\circ}$ while one wall of the chamber was Plexiglas to allow video recording. One milliliter of vanilla extract was placed in a small Petri dish below the cage flooring and out of the animals reach, also to enhance the salience of the context. Mice were allowed to explore the chamber for $3 \mathrm{~min}$. During this time, activity was scored to control for possible locomotor differences between wild-type and mutant mice by counting the number of crossings through the midline of the chamber and the number of rearings performed. After $3 \mathrm{~min}$, an $80-\mathrm{dB}$ tone $(2 \mathrm{kHz})$ was played for $20 \mathrm{~s}$. During the last second of the tone, a mild electric current $(0.70 \mathrm{~mA})$ was passed through the flooring with a 1-s duration. Tone and footshock delivery were controlled by software (Lab Linc Operant Control Software; Coulbourne Instruments). Following a 1-min interval, the tone was played again for $20 \mathrm{~s}$ accompanied by a 1-s current stimulus overlapping the last second of the tone. After another 1-min interval, the toneshock sequence was repeated one last time for a total of three pairings, and the mice were left in the chamber for another 30 s. The chamber was cleaned with $70 \%$ ethanol between individual sessions. Twenty-four hours later, on day 2 , mice were evaluated for fear conditioning to context by reintroducing them to the conditioning chamber for $8 \mathrm{~min}$ without tone or footshock, and freezing behavior was scored (context test). Freezing behavior is defined here as the lack of all body movement except for that associated with respiration. Animals were observed every $10 \mathrm{~s}$, at which time the animal was either considered freezing or not. The number of observations during which the animal was freezing was divided by the total number of observations made to determine the percent freezing. Forty-eight hours after the initial conditioning, on day 3 , mice were evaluated for cued fear conditioning to the tone (tone test). Mice were placed in a novel chamber $(12 \times 8 \times 5$ in. $)$ that was cleaned between individual sessions with $70 \%$ isopropyl alcohol. Following 3 min of exploration, an $80-\mathrm{dB}, 2-\mathrm{Hz}$ tone was played for $8 \mathrm{~min}$, and behavior was observed and recorded every $10 \mathrm{~s}$ for the duration of the tone.

Y-maze

Spontaneous spatial novelty preference was assessed. This test assesses rapidly acquired, short-term spatial memory and relies on the fact that normal mice prefer novel over familiar spatial environments. These mice had no previous experience of any maze testing or of the laboratory in which the experiment was conducted. The spontaneous spatial novelty preference test was conducted using a Y-maze constructed from transparent Perspex. Each arm was $30 \mathrm{~cm}$ long and $8 \mathrm{~cm}$ wide, with 20-cm-high walls. Mice were assigned two arms ("start arm" and "other 
arm"), to which they were exposed during the first phase of the test (the exposure phase). During this exposure phase, the entrance to the third arm of the maze (the "novel arm" during the subsequent test phase) was blocked off with a sheet of opaque Perspex. For the exposure phase, mice were placed at the end of the start arm and were allowed to explore the start arm and the other arm for $5 \mathrm{~min}$ (beginning from the time the mouse first left the start arm). Entry into an arm was defined when a mouse placed all four paws into an arm, and similarly a mouse was considered to have left an arm if all four paws were placed outside that arm. The mouse was then removed from the maze and returned to its home cage for $1 \mathrm{~min}$. The mouse was then returned to the maze for the test phase, during which it now had access to all three arms of the maze. The mouse was again placed at the end of the start arm and allowed to explore for $2 \mathrm{~min}$. The amount of time the mouse spent in each of the arms of the maze and the number of entries into each arm were recorded during both the exposure phase and the test phase. For the test phase, a discrimination ratio [novel arm/(novel other arm)] was calculated for both arm entries and time spent in arms.

\section{Statistical Analysis}

Statistical analysis was performed using one-way ANOVA, followed by the Bonferroni test (for multiple factors). Alternatively, Student's $t$ test was applied to data with multiple variables and one factor. Normalized FRET results were obtained from multiple sister slices, and their means were calculated ( $n=4-6$ slices with a total of 8-16 readings). $p$ value of $<0.05$ was selected to indicate statistical significance.

\section{Results}

Cultured primary neurons and neurons in vivo are not at steady state or at dynamic equilibrium (Levine 2005); therefore, it is critical to be able to understand how individual neurons respond and behave in real time to stimuli by monitoring single-cell behavior. Traditional assays, including flow cytometry, enzyme-linked immunoabsorbent assays and biochemistry assays can only measure neuronal behavior under static conditions and are limited to measuring the activity in the bulk environment of a group of cells (Farr et al. 2010). We previously developed a FRET-based single-cell sensor capable of monitoring calpain activation in single neuronal cells in real time (Zadran et al. 2010a, b). Utilizing this sensor, we discovered a calcium-independent mechanism for neuronal calpain2 activation by brain-derived neurotrophic factor (BDNF) and estrogen through ERK-mediated phosphorylation.
Additionally, calpain 2 activation resulted in both increased actin polymerization in dendritic spine-like structures following BDNF treatment, and increased GluR1 subunits of AMPA receptors following estrogen treatments in vitro (Zadran et al. 2009, 2010b). Here, we developed a FRETbased animal model, Calpain Activation by Fluorescence Imaging (CAFI), to monitor spatio-temporal localization of in vivo calpain activation in single cells and in real time (Stockholm et al. 2005).

To ensure that CAFI mice were a reliable in vivo neuronal calpain activation reporter, CAFI mice were subjected to Fear-Conditioning assay to ensure they did not exhibit deficits in learning and memory; CAFI mice performance in fear conditioning was similar to that of wildtype mice (Supplemental Figure 1). Subsequently, acute hippocampal slices were prepared from young adult CAFI mice (6-8 weeks). Slices were incubated in aCSF for $1 \mathrm{~h}$ in the dark prior to confocal or spectrofluorometric analysis to remove residual FRET loss from calpain activation during acute slice preparation. Slices were then incubated with or without calpain inhibitor for $20 \mathrm{~min}$ prior to neural stimulation. Neural stimulation included both electrophysiology (theta burst stimulation or TBS) and chemically induced stimulation with BDNF or tetraethylammonium (TEA), a potassium channel blocker. Both BDNF- and TEA-treated slices showed a significant loss of FRET (Fig. 1a); FRET loss was not observed in slices pretreated with calpain inhibitor III. Confocal images of single neurons in hippocampal CA1 field displayed FRET loss in dendrites within only $10 \mathrm{~min}$ of neural stimulation. Interestingly, neuronal cells within the CA1 field displayed differential calpain activation; several neuronal cells displayed little or no FRET loss, while others showed significant FRET loss, particularly in dendritic spines within only 5 min of neural stimulation (Fig. 1c). The CA1 field of hippocampal slices was then microdissected out and homogenized, and bulk cell FRET behavior was quantified via spectrofluorometry. CA1 homogenates from BDNF-, TEA- or TBS-treated slices also displayed calpain-dependent FRET loss, with TBS-treated slices eliciting the greatest calpain activation (Fig. 1b).

We also previously developed a rhodamine-phalloidin fluorescence enhancement calpain functional assay to monitor both the rate and the degree of calpain-initiated actin polymerization in single cells in vitro (Zadran et al. 2009, 2010b). We modified this assay to monitor in vivo calpain-mediated actin polymerization in single neuronal cells in CA1 field of acute hippocampal slices from C57BL/6 wild-type mice. BDNF- and TEA-treated as well as TBS-stimulated slices showed increased actin polymerization in a calpain-dependent manner, as observed by confocal microscopy (Fig. 1d). Differential calpaindependent actin polymerization was also monitored in CA1 
A
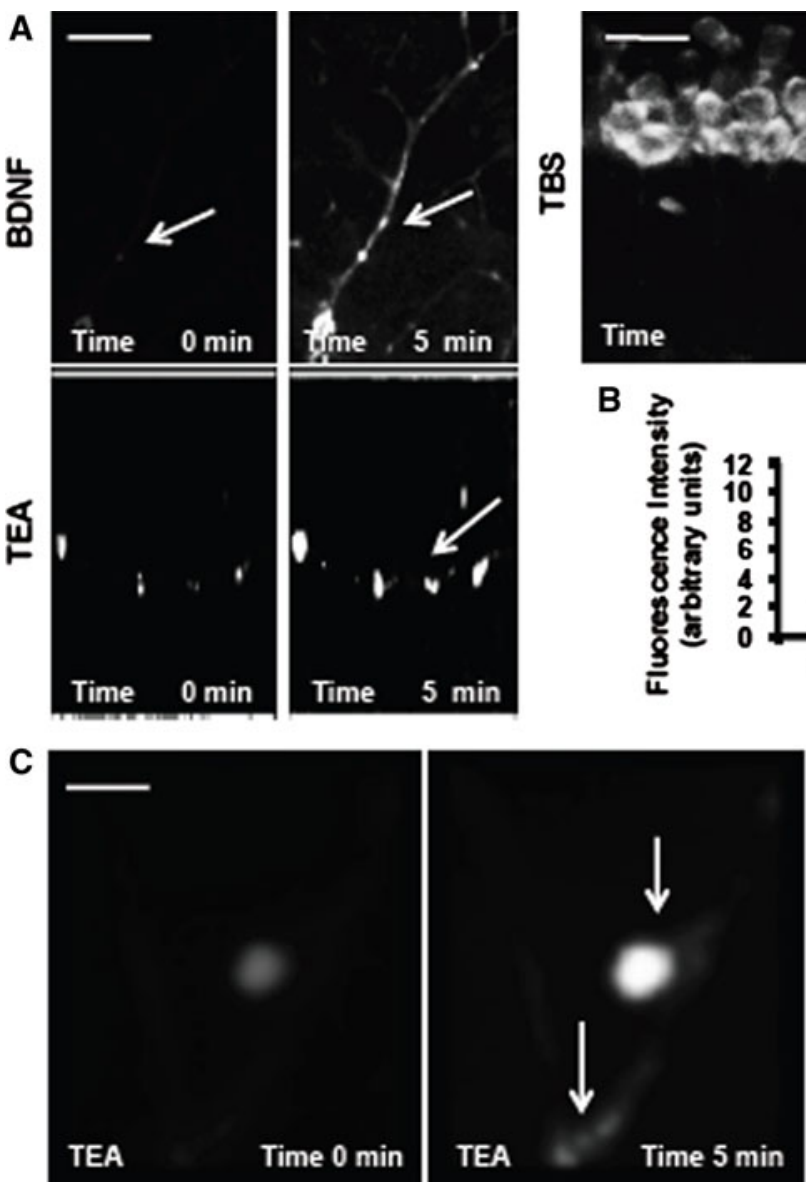

D
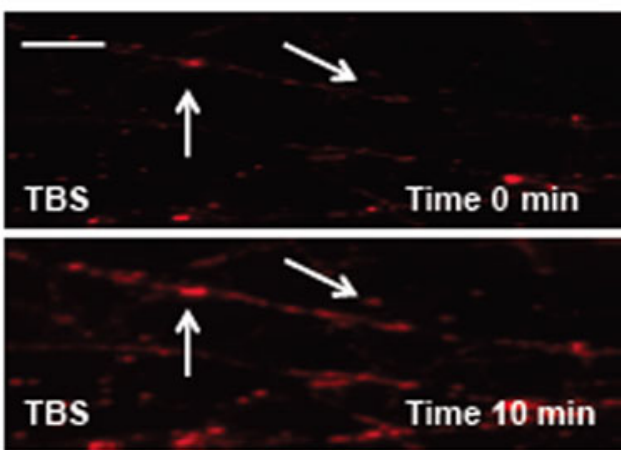

Fig. 1 Neuronal stimulation activates calpain in single neurons in region CA1 of acute hippocampal slices. a A dendritic branch was selected for imaging before the application of BDNF $(50 \mathrm{ng} / \mathrm{ml})$ or TEA $(10 \mathrm{mM})$. Confocal images $(65 \mathrm{X})$ were taken again $5 \mathrm{~min}$ after the treatment and clearly indicate localized FRET loss in cell bodies as well as in dendrites and spine-like puncta. Similarly, TBS stimulation also resulted in differential FRET loss in CA1 pyramidal neurons, their proximal dendrites and spine-like structures after 5 min. Scale bar $=20 \mu \mathrm{m}$. b Field CA1 was microdissected following slice treatment, and FRET was analyzed with spectrofluorometry (mean \pm SD of 5 experiments; $* p<0.01$ ). c To monitor calpain activation in vivo in single cells in real time, a dendritic branch was selected for imaging before the stimulation with TEA. Confocal images $(65 \mathrm{X})$ were taken again $5 \mathrm{~min}$ after treatment and clearly indicate FRET loss. Scale bar $=20 \mu \mathrm{m}$. d Actin polymerization in acute hippocampal slices from wild type C57BL/6 mice was monitored via confocal microscopy $(65 \mathrm{X})$ following TBS, scale bar $=50 \mu \mathrm{m}$. Actin polymerization was analyzed in field CA1 with spectrofluorometric analysis following treatment with BDNF or TEA and TBS (mean \pm SD of four experiments, ${ }^{*} p<0.01, * * p<0.05$ ) field. Neuronal cells that did exhibit increased actin polymerization had it heavily localized in dendritic spine-like structures.

To monitor the calpain isoform-specific effects on synaptic plasticity and learning and memory in vivo, we developed a novel vector that consisted in coupling a calpain2 siRNA to the rabies virus glycoprotein (RVG) peptide using a chimeric peptide consisting of nine arginine residues. RVG has been shown to enable transvascular delivery, specifically to the brain by binding to acetylcholine receptors (Rohn et al. 2012). We have also previously used heptarginine motifs to successfully deliver peptides into hippocampal neurons without the need for transfection reagents (Zadran et al. 2009, 2010b). We first tested the efficacy of the RVG-calpain2 siRNA construct in primary hippocampal cultures (Fig. 2a). A significant decrease in calpain2 protein levels was observed following $24 \mathrm{~h}$ of incubation with the construct. The RVG construct was then injected intravenously in the tail of young adult C57BL/7 mice (6-8 weeks) daily for 4 days. Brain levels of calpain2 were analyzed on day 5 to ensure sufficient gene silencing. Western blot analysis showed a significant decrease in calpain2 levels in whole brain homogenates of RVG-construct-treated mice (Fig. 2a). We also monitored primary hippocampal neurons for cytotoxicity due to the RVG-calpain2 siRNA construct using a MTT assay and observed no significant cytotoxicity due to treatment (Supplemental Figure 2A). To monitor the specificity of the RVG construct to brain calpain2, calpain2 levels in the mouse heart and liver were also examined via Western 
blotting. No significant affect on calpain2 levels was observed in those regions (Supplemental Figure 2B).

To examine whether RVG-construct-mediated gene silencing of calpain 2 resulted in deficits in synaptic plasticity and in learning and memory, C56BL/6 mice (6-8 weeks) were injected intravenously daily for 4 days (Fig. 2b), and hippocampal slices were prepared for electrophysiological assessment of long-term potentiation (LTP) of synaptic transmission in field CA1 (Fig. 2c). LTP was completely abolished in mice treated with the RVGcalpain2 siRNA construct $(103 \pm 8 \%, n=3$, as compared to scrambled control: $174 \pm 10 \%, \quad n=4$; $p<0.002)$; in contrast, paired-pulse facilitation and I/O curves were not affected as compared to animals injected for 4 days with a RVG construct with a scrambled siRNA (not shown). Another set of mice treated with the same constructs was tested for their performance in a Y-maze. Mice treated with the RVG-calpain2 siRNA construct exhibited a significant decrease in spontaneous alternation, implicating for the first time a key role for the calpain isoform, calpain2, in learning and memory (Fig. 2d). No changes in Y-maze learning was observed in mice injected with the RVG construct coupled to a scrambled siRNA.

\section{Discussion}

While the hypothesis that calpain activation plays a critical role in synaptic plasticity and learning and memory was published almost 30 years ago, it has been difficult to provide convincing evidence to support it and to identify potential calpain substrates that could account for the synaptic modifications associated with LTP and learning and memory. Technical difficulties in effectively monitoring calpain activation at individual synapses in real time and in down-regulating calpain have been a significant obstacle for understanding the molecular and cellular mechanisms underlying synaptic plasticity. To this end, we utilized a calpain activation mouse model to monitor calpain activation in vivo via FRET kinetic analysis, and a

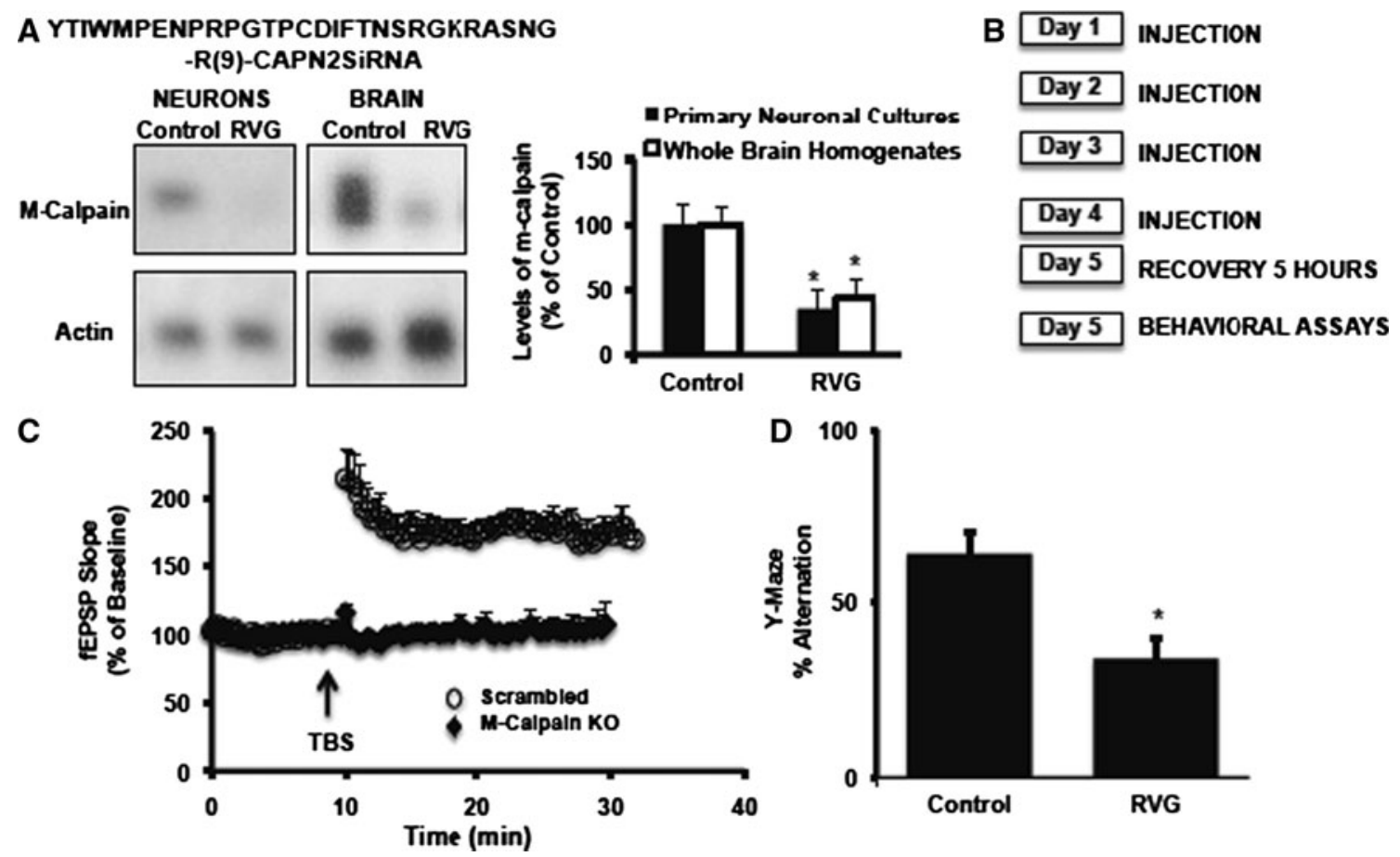

Fig. 2 Effects of RVG-siRNA-calpain2 constructs on LTP and performance in the Y-maze a Primary hippocampal cultures (DIV14) and C57BL/6 mice were treated with the RVG-siRNA construct. Levels of calpain2 were analyzed by Western blots; Western blots were stripped and reanalyzed for actin levels. Homogenates from primary neurons following $24 \mathrm{~h}$ of incubation with the RVG construct. Whole brain homogenates from C57BL/7 mice (6-8 weeks) injected with the RVG-siRNA construct daily (iv) for 4 days. Quantification of Western blots similar to those shown. Results were normalized to the levels of actin and expressed as percent of saline-treated or saline-injected controls and are mean $\pm \mathrm{SD}$ of five experiments; ${ }^{*} p<0.05$. b RVG injection timetable. c Effect of RVG-siRNA-calpain2. C57BL/7 mice (6-8 weeks) were injected with the RVG-siRNA constructs daily (iv) for 4 days. One day after the last injection, slices were prepared for electrophysiology. LTP was elicited by theta burst stimulation (TBS) (five bursts of four stimuli delivered at $100 \mathrm{~Hz}$ repeated at $5 \mathrm{~Hz}$ ). Slope of EPSPs was measured and results normalized to the average value measured during the $10 \mathrm{~min}$ baseline period. Results are mean \pm SD of 3-4 slices from two and three mice. d Percent alternation in the Y-maze in control and RVG-treated mice targeting calpain2 (mean $\pm \mathrm{SD}$ of 7 experiments, $* p<0.05$ ) 
novel RVG-coupled calpain2 siRNA vector to down-regulate calpain in order to elucidate the role of calpain in synaptic plasticity (LTP) and learning and memory.

FRET is a highly sensitive technique that provides both spatial and temporal analysis of molecular events. The CAFI mouse model utilizes a traditional CFP and YFP FRET pair linked with a peptide specific for calpain, corresponding to the amino acid sequence of the calpain cleavage site of spectrin. FRET was analyzed and quantified with both a scanning confocal microscope and a spectrofluorometer. In agreement with the results we obtained using a single cell calpain activation FRET probe for analysis in cultured neurons (Zadran et al. 2009), calpain activation in hippocampal slices prepared from CAFI mice resulted in FRET loss and increased donor fluorescence emission.

Dendritic spines are highly dynamic structures that continuously undergo changes in shape and size as a result of synaptic activity. The current classification of spines is based on the relative size and shape of the spine head and neck. Mushroom spines have a large spine head and a narrow neck, are selectively enriched in F-actin and polyribosomes, and have well-developed calcium-handling machinery (Zadran et al. 2010a). Activation of both NMDA and AMPA receptors (GluR) participates in spine formation and regulates spine maturation and spine dynamics in part via calcium-dependent regulation of F-actin turnover. It is now generally admitted that LTPinducing stimuli produce long-lasting changes in synaptic size and structure that are correlated with synaptic strength (Zadran et al. 2010a).

We previously reported no deficits in synaptic plasticity and learning behavior in calpain1 knockout mice, suggesting that calpain2 might be the calpain variant involved in mechanisms of synaptic plasticity (Zadran et al. 2010a). To further address this issue, we generated a novel RVGcoupled calpain2 siRNA vector that was capable of selectively reducing levels of calpain 2 in the brain following systemic injection. The vector required multiple treatments over a course of several days to significantly reduce brain calpain levels. Animals treated with the vector exhibited marked impairment in LTP induction in field CA1 of hippocampal slices and significant deficits in Y-maze learning, as compared to animals treated with the control vector, indicating that calpain 2 activation is a necessary event in synaptic plasticity and in hippocampus-dependent learning and memory.

Almost thirty years ago, Lynch and Baudry proposed that calpain-mediated modifications of dendritic spine structure and function were a key element in the cellular processes underlying certain forms of learning and memory in mammalian brain. Recently, several studies have revealed critical features about the calpain system illuminating new mechanisms of calpain activation in the central nervous system (CNS) and revitalizing the debate regarding the role of calpain in learning and memory. Progress in our understanding of complex phenomena such as synaptic plasticity and learning and memory depends on the development of new tools that can provide insight into the precise temporal and spatial sequence of molecular events underlying them. Here, we develop two novel technologies: an animal model to monitor differential in vivo calpain activation in single cells in real time and an RVG-targeting construct capable of calpain isoform-specific gene silencing in vivo. The application of these technologies identified for the first time calpain 2 activation as a necessary event in synaptic plasticity and in learning and memory. These technologies not only provide information regarding the nature of calpains in the brain but also are critical for the development of therapeutics.

Acknowledgments We would like to express our gratitude to Drs. Haijiang Cai, Qingyu Qin and Xiaoning Bi for providing assistance in imaging and electrophysiology experiments. We are also grateful to Ariana Chehrazi, Georgina Smith, Lauren Alderette, James Bowman III and Maria Agosto for technical assistance. This work was supported by grants from the National Institute of Neurological Disorders and Stroke (USC NINDS 5R01NS057128-03 to Dr. M Baudry). Dr. Sohila Zadran was supported by a National Institute of Health T32 Post-Doctoral Fellowship (Caltech NIH 5 T32NS7251-24 to Dr. $\mathrm{R}$ Andersen). Homera Zadran was supported by the National Science Foundation Graduate Research Fellowship Program (NSF GRFP).

Conflict of interest The authors declare no competing financial interests.

\section{References}

Camins, A., Verdaguer, E., Folch, J., \& Pallàs, M. (2006). Involvement of calpain activation in neurodegenerative processes. CNS Drug Reviews, 12(2), 135-148.

Chan, S. L., \& Mattson, M. P. (1999). Caspase and calpain substrates: Roles in synaptic plasticity and cell death. Journal of Neuroscience Research, 58(1), 167-190.

Chen, L. Y., Rex, C. S., Casale, M. S., Gall, C. M., \& Lynch, G. (2007). Changes in synaptic morphology accompany actin signaling during LTP. Journal of Neuroscience, 20, 5363-5372.

Czogalla, A., \& Sikorski, A. F. (2005). Spectrin and calpain: A 'target' and a 'sniper' in the pathology of neuronal cells. Cellular and Molecular Life Sciences, 62(17), 1913-1924. (Review).

Demarchi, F., \& Schneider, C. (2007). The calpain system as a modulator of stress/damage response. Cell Cycle, 6(2), 136-8. (Epub 2007 Jan 27. Review).

Donkor I. O. (2011). Calpain inhibitors: A survey of compounds reported in the patent and scientific literature. Expert Opinion on Therapeutic Patents, 21(5), 601-36. (Epub 2011 Mar 24. Review).

Farr, C., \& Berger, S. (2010). Measuring calpain activity in fixed and living cells by flow cytometry. Journal of Visualized Experiments, (41). doi:10.3791/2050. 
Goll, D. E., Thompson, V. F., Li, H., Wei, W., \& Cong, J. (2003). The calpain system. Physiological Reviews, 3, 731.

Grammer, M., Kuchay, S., Chisht, A., \& Baudry, M. (2003). Lack of phenotype for LTP and fear conditioning learning in calpain 1 knock-out mice. Neurobiology of Learning and Memory, 3, 222-227.

Greget, R., Pernot, F., Bouteiller, J. M., Ghaderi, V., Allam, S., Keller, A. F., Ambert, N., Legendre, A., Sarmis, M., Haeberle, O., Faupel, M., Bischoff, S., Berger, T. W., Baudry, M. (2011). Simulation of postsynaptic glutamate receptors reveals critical features of glutamatergic transmission. PLoS One, 6(12), e28380. (Epub 2011 Dec 15).

Huang, Y., \& Wang, K. K. (2001). The calpain family and human disease. Trends in Molecular Medicine, 7(8), 355-362. (Review).

Levine, R. D. (2005). Molecular reaction dynamics. Cambridge, UK: Cambridge University Press.

Liu, J., Liu, M. C., \& Wang, K. K. (2008). Calpain in the CNS: From synaptic function to neurotoxicity. Science Signal, (14):re1 (Review).

Lynch, G., \& Baudry, M. (1984). The biochemistry of memory: A new and specific hypothesis. Science, 224, 1057-1063.

Rohn, S., Suttkus, A., Arendt, T., \& Ueberham, U. (2012). RVG peptide as transfection reagent for specific cdk4 gene silencing in vitro and in vivo. Journal of Drug Targeting, 20(4), 381-388.

Sorimachi, H., Hata, S., \& Ono, Y. (2011). Calpain chronicle-An enzyme family under multidisciplinary characterization. Proceedings of the Japan Academy. Series B, Physical and biological sciences, 87(6), 287-327.
Stockholm D, Bartoli M, Sillon G, Bourg N, Davoust J, Richard I. (2005). Imaging calpain protease activity by multiphoton FRET in living mice. Journal of Molecular Biology, 346(1), 215-22. (Epub 2004 Dec 16).

Takano, J., Mihjra, N., Fujioka, R., Hosoki, E., Chishti, A. H., \& Saido, T. C. (2011). Vital role of the calpain-calpastatin system for placental-integrity-dependent embryonic survival. Molecular and Cellular Biology, 31(19), 4097-4106.

Verity, M. A. (1992). Ca(2+)-dependent processes as mediators of neurotoxicity. Neurotoxicology, 13(1), 139-147. Review.

Vosler, P. S., Brennan, C. S., Chen, J. (2008). Calpain-mediated signaling mechanisms in neuronal injury and neurodegeneration. Molecular Neurobiology, 38(1), 78-100. (Epub 2008 Aug 7. Review).

Zadran S., Bi X., \& Baudry M. (2010a). Regulation of calpain-2 in neurons: Implications for synaptic plasticity. Molecular Neurobiology, 42(2), 143-50. (Epub 2010 Oct 6. Review).

Zadran, S., Jourdi, H., Rostamiani, K., Qin, Q., Bi, X., \& Baudry, M. (2010b). Brain-derived neurotrophic factor and epidermal growth factor activate neuronal $\mathrm{m}$-calpain via mitogen-activated protein kinase-dependent phosphorylation. Journal of Neuroscience, 3, 1086-1095.

Zadran, S., Qin, Q., Bi, X., Zadran, H., Kim, Y., Foy, M. R., et al. (2009). 17- $\beta$-Estradiol increases neuronal excitability through MAP kinase-induced calpain activation. Proceedings of the National Academy of Sciences of the United States of America, 51, 21936-21941. 